


\section{La Cátedra de Arquitectura Sacra en la Facultad de Arquitectura y Urbanismo de la Universidad Católica de La Plata}

Course in Sacred Architecture - Architecture and Urban Planning Department - Catholic University of La Plata

Federico G. Gottfried · Facultad de Arquitectura y Urbanismo. Universidad Católica de La Plata (Argentina)

\section{RESUMEN}

En 1987, Juan Pablo II visita la Argentina y pide a los laicos que se comprometan con la educación y la cultura. Con la anuencia de monseñor Antonio Quarracino, arzobispo de La Plata, se crea la Cátedra de Arquitectura Sacra en la carrera de Arquitectura de la UCALP. El desafío fue —y sigue siendo- que los alumnos comprendan las necesidades del espacio sacro católico, reforzando la doctrina y los conceptos arquitectónicos. Mediante un programa que se perfeccionó a lo largo de los años, se aborda la investigación, el estudio, la reflexión, el acompañamiento docente y el hacer arquitectura. Todo ello tiene como objetivo que el alumno, alumbrado por el Evangelio, logre diseñar un edificio con la comprensión cabal del concepto iglesia. Esta búsqueda académica y personal de los educandos se refleja en anteproyectos de arquitectura construibles, que sintetizan la impronta estética y resolutiva de cada uno de ellos.

\section{PALABRAS CLAVE}

Arquitectura sacra, educación, iglesia, Universidad Católica de La Plata, Argentina.

\section{ABSTRACT}

In 1987, John Paul II visits Argentina and calls on the laity to commit to education and culture. With the blessing of Monsignor Antonio Quarracino, archbishop of La Plata, the chair of Sacred Architecture in the UCALP career of Architecture is created. The challenge was - and still remains - that students understand the needs of Catholic sacred space, reinforcing their doctrine and architectural concepts. Using a program that was perfected over the years, research, study, reflection, teaching and doing architecture accompaniment is addressed. The aim of all this is that the student, enlightened by the Gospel, be able to design a building with a full understanding of the concept church. This academic and personal search of learners is reflected in draft constructible architecture, which synthesize aesthetics and decisive imprint of each of them.

\section{KEYWORDS}

Sacred Architecture, Education, Church, Catholic University of La Plata, Argentina. 
Con motivo de la visita de Su Santidad Juan Pablo II a la Argentina en 1987, durante sus homilías, el Santo Padre reiteró su pedido a los laicos sobre la responsabilidad de actuar en la educación y la cultura. Surgió entonces la idea de los arquitectos Alejandro Scaserra y Federico G. Gottfried, con el aval de monseñor Antonio Quarracino, entonces arzobispo de La Plata, de incorporar la materia Arquitectura Sacra en la carrera de arquitectura de la UCALP.

Esta cátedra, que se encuentra dentro del Departamento de Teología, fue creada con la intención de reforzar el aspecto doctrinal y arquitectónico de los estudiantes, sobre la idea de conocer la razón de ser del edificio iglesia y los componentes que lo conforman.

Esta síntesis, dirigida hacia el conocimiento del espacio sacro, produce un camino inverso en la tarea del arquitecto, ya que el comitente es quien tiene un valor superlativo, por ser el mismo Dios. De allí la importancia de transitar este camino en humildad, para comprender que lo más valioso de la iglesia es lo que contiene: la presencia del Dios vivo.

\section{INTERVENCIONES EN IGLESIAS EXISTENTES}

El primer desafío a enfrentar, respecto a las intervenciones en las iglesias tradicionales, era el tema del altar frente al pueblo. El problema a solucionar era la ubicación del sacerdote, al quedar físicamente entre los dos altares y, consecuentemente, dar la espalda al sagrario, ubicado sobre el altar originario. Una posible solución, respetando el eje altar/sacerdote/sede/sagrario, fue elevar el sagrario hasta una altura conveniente, provocando escalones para llegar hasta ese sitio, y aprovechando el muro de contención de los mismos como respaldo para la sede.

Como modo de integrar al sacerdote con la asamblea, en muchas iglesias tradicionales los arquitectos en general decidieron retirar el comulgatorio, una decisión un tanto controvertida, ya que la altura del mismo no impedía una buena relación visual entre ambos.

Respecto a la incorporación del ambón, en muchos casos y siguiendo una misma estética que el altar cara al pueblo, entendíamos que era importante respetar el antiguo púlpito, ya que formaba parte de la historia del edificio.

En cuanto a la ubicación del baptisterio, cercana al presbiterio, y próxima a la asamblea, exigía un diseño de un subespacio, al igual que los confesionarios, debi- do a las particulares relaciones que se daban entre los fieles y el sacerdote.

La ubicación del coro, que dejaba su tradicional ubicación sobre un nivel superior en la entrada de la iglesia, ofrecía una alternativa: la ubicación opuesta al baptisterio, adelante, en un primer nivel. Esto favorecía su función por la reconocida calidad acústica que presentan las iglesias antiguas hacia la asamblea. La capilla de San José, en la localidad de Bernal, ofrece estas opciones (Fig. 01-03).

Existen otras variantes, como la de colocar el sagrario en una capilla del Santísimo o la de trasladar la sede hacia un costado del presbiterio. De esta manera, entendemos que la dimensión del edificio ofrece una cantidad de posibilidades muy variadas y específicas para cada una de ellas.

\section{LAS IGLESIAS NUEVAS}

El segundo reto consistía en comprender el sentido de la asamblea y su ubicación frente al presbiterio, con una gran variedad de propuestas.

La tradicional planta en cruz latina cedía ante la planta en cruz griega, al ofrecer una ubicación de la asamblea con mayores beneficios visuales, al igual que las plantas en forma de elipse, círculo y otras soluciones geométricas similares.

En el año 1993, la Conferencia Episcopal Argentina produjo los Lineamientos generales para la construcción de iglesias, donde trataba de reflejar la doctrina y las recomendaciones de la Iglesia a la luz de las disposiciones emanadas del Concilio Vaticano II y las recientes modificaciones que presentaba el Código de Derecho Canónico (1983), actualmente vigente. En estos lineamientos generales se afirma: «La sacralidad, como una cualidad del espacio religioso, queda formalmente definida cuando el templo es consagrado o bendecido. Así como toda consagración segrega algo o alguien para ofrecerlo a Dios, así también el espacio donde tendrá lugar la actividad religiosa queda, por la consagración o bendición, separado de otro lugar y destinado exclusivamente para el culto, del mismo modo que el cristiano cuando es consagrado por el bautismo, la confirmación o el orden sagrado, aparece como estando en el mundo sin ser del mundo» (7-8).

Estos conceptos, aplicados a la arquitectura, los podemos observar en dos obras paradigmáticas situadas en la provincia de Buenos Aires. 


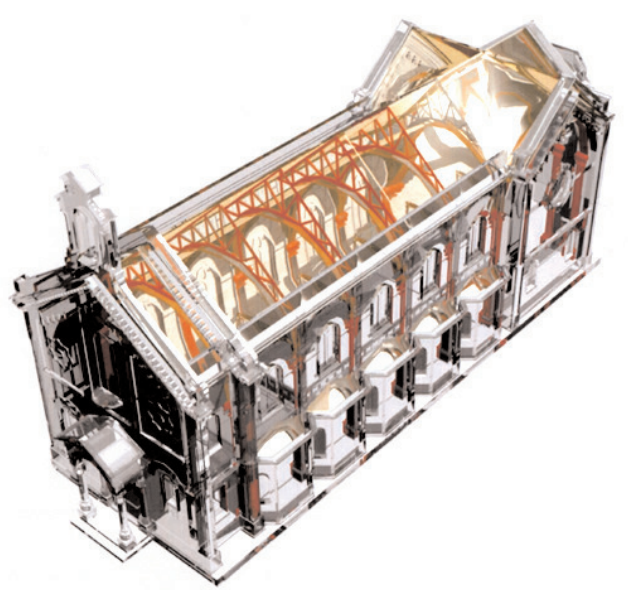

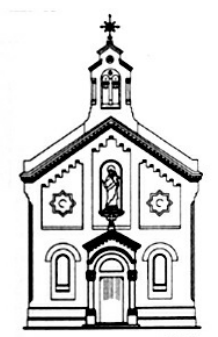

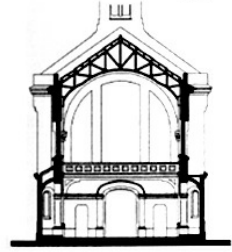

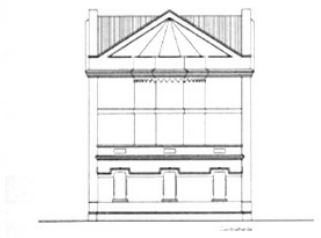

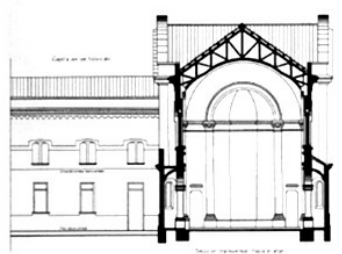

Fig. 01. Federico G. Gottfried, Capilla de San José, Bernal (Buenos Aires); perspectiva estructural, espacial y constructiva, 2009.

Fig. 02. Federico G. Gottfried, Capilla de San José, Bernal (Buenos Aires); estructura, vista, corte, 2009.

Fig. 03. Federico G. Gottfried, Capilla de San José, Bernal (Buenos Aires); módulos espaciales y elementos simbólicos e históricos, 2014.

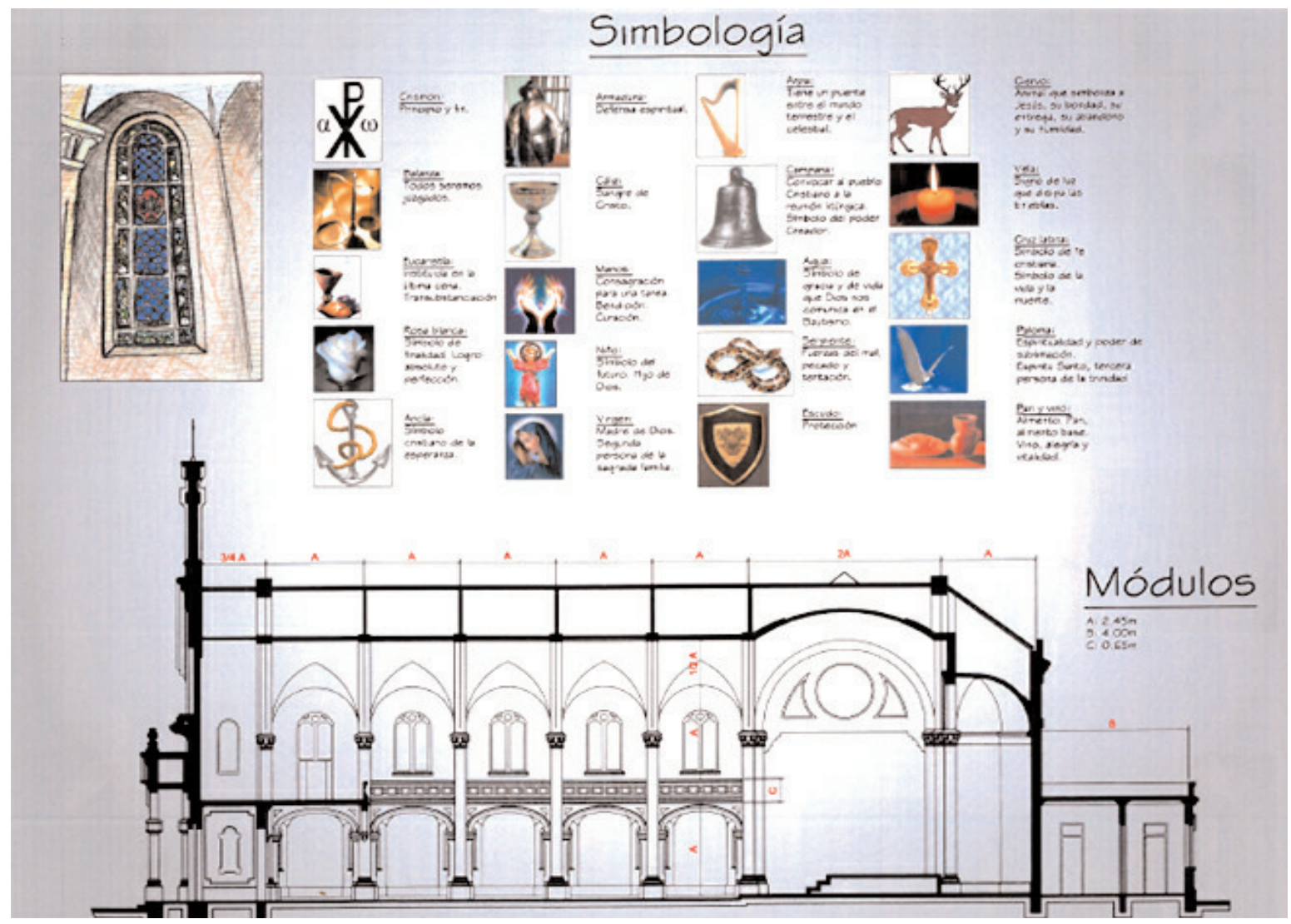



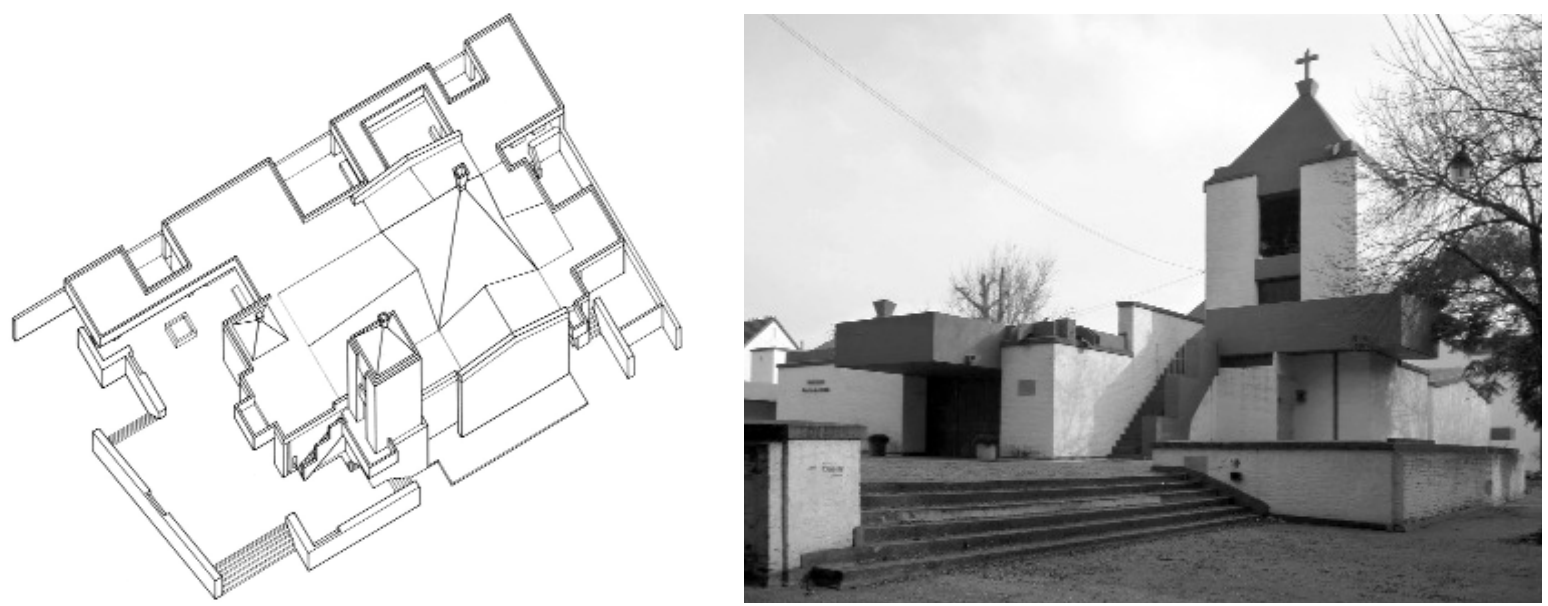

Fig. 04 y 05. Claudio Caveri y Federico Ellis, Nuestra Señora de Fátima, Martínez (Buenos Aires), 1956/58.

Una es la iglesia de Nuestra Señora de Fátima, en la ciudad de Martínez, partido de San Isidro, de los arquitectos Caveri y Ellis, que trata de reinterpretar el estilo histórico colonial con elementos vanguardistas del momento. Este estilo, que marca una época en la arquitectura argentina, se lo conoce como casablanquismo, entre otras cosas, por el color de sus muros (Fig. 04-05).

La otra es la iglesia San Gabriel Arcángel, en la localidad de Adrogué, obra realizada por los arquitectos Mario González y Raquel Adesso. En ella se desmaterializa la planta en cruz y se explica la posición del altar como un centro de espirales logarítmicas en progresión con la proporción áurea (Fig. 06-07).

\section{EL PROGRAMA DEL CURSO}

Por último, el tercer desafío correspondió a lograr en los alumnos un proceso cuyo fin sea la elaboración de un anteproyecto de una iglesia parroquial. A este objetivo se llegó, y se logra en cada año que se cursa la materia. Para ello, se les propone realizar el análisis de determinadas iglesias y responder a una serie de cuestionarios para evaluar su nivel de comprensión del espacio sacro. Asimilados los distintos aspectos propios del objeto de estudio, se definen los pasos a seguir respecto a una metodología de diseño. Esto es controlado por una suma de conceptos, ideas, esquemas, y correcciones que deben constituir los elementos que confor- man una carpeta de proceso continuo que se trabaja durante el curso. La materia consta de un programa que aborda tres unidades.

Unidad 1. Investigación, estudio, reflexión.

-Ubicación espacio/tiempo.

-El hecho arquitectónico: aspectos funcional, fomal, espacial, tecnológico, táctico, semántico y estético. -Relaciones urbanas.

-Conclusión.

-Propuesta.

\section{Unidad 2. Docencia}

-El legado de la tradición arquitectónica.

-El templo cristiano.

-Conceptos de lo sacro.

-El espacio sagrado.

-Los imperativos de la liturgia.

-Configuración del espacio sagrado.

-Emplazamiento de la iglesia.

-Condicionamientos técnicos.

-Etapas evolutivas de la arquitectura contemporánea. -La arquitectura sacra como reunión de artes y artesanías.

-La música sacra.

\section{Unidad 3. El hacer}

- Anteproyecto.

- Bibliografía. 

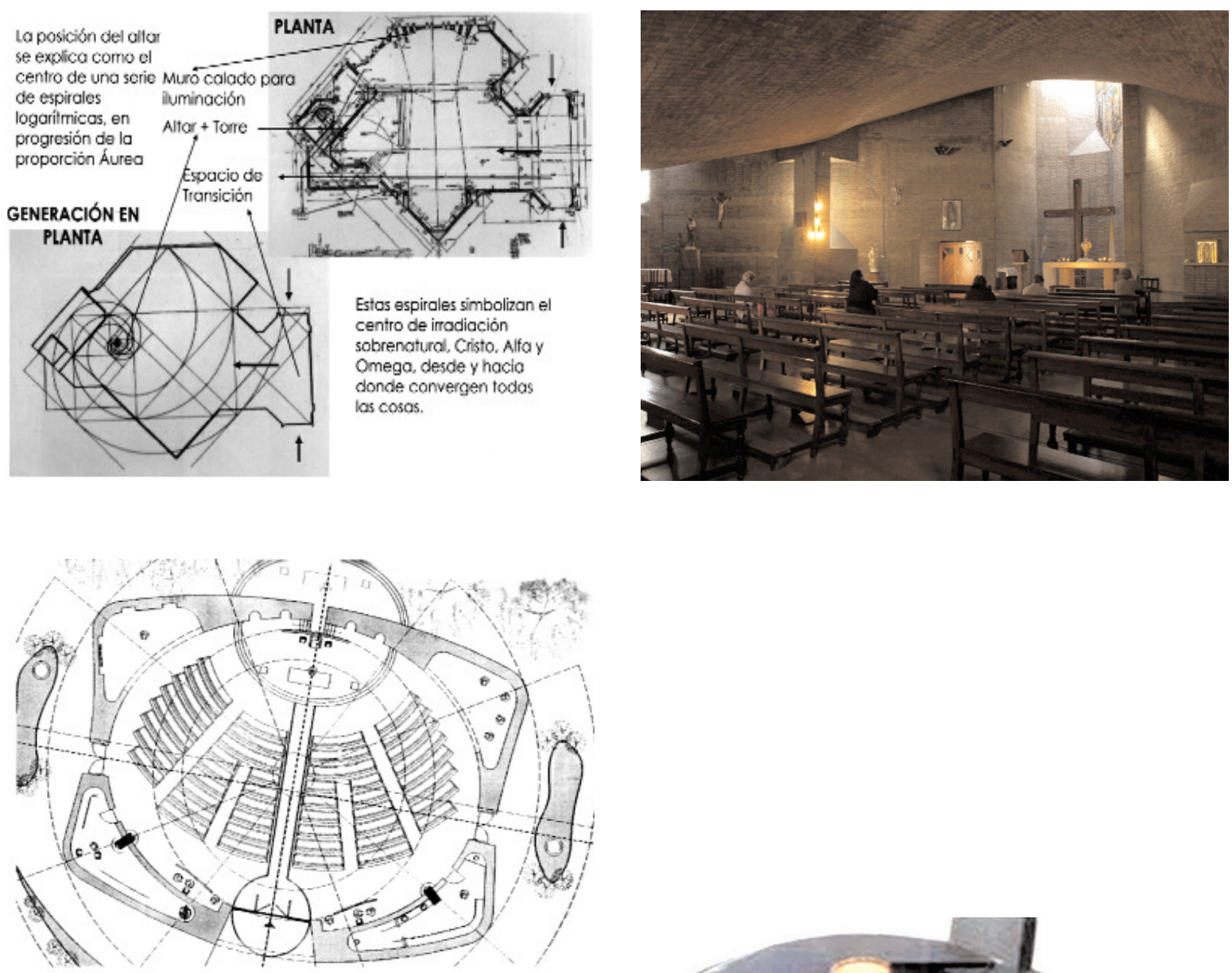

Fig. 06 y 07. Mario González y Raquel Adesso, San Gabriel Arcángel,

Adrogué (Buenos Aires), 1979; gráficos de estudio sobre el plano.

Fig. 08 y 09. Mariel Eliana Trotta, anteproyecto para una iglesia en Quilmes (Buenos Aires), 2012; planta y perspectiva seccionada.

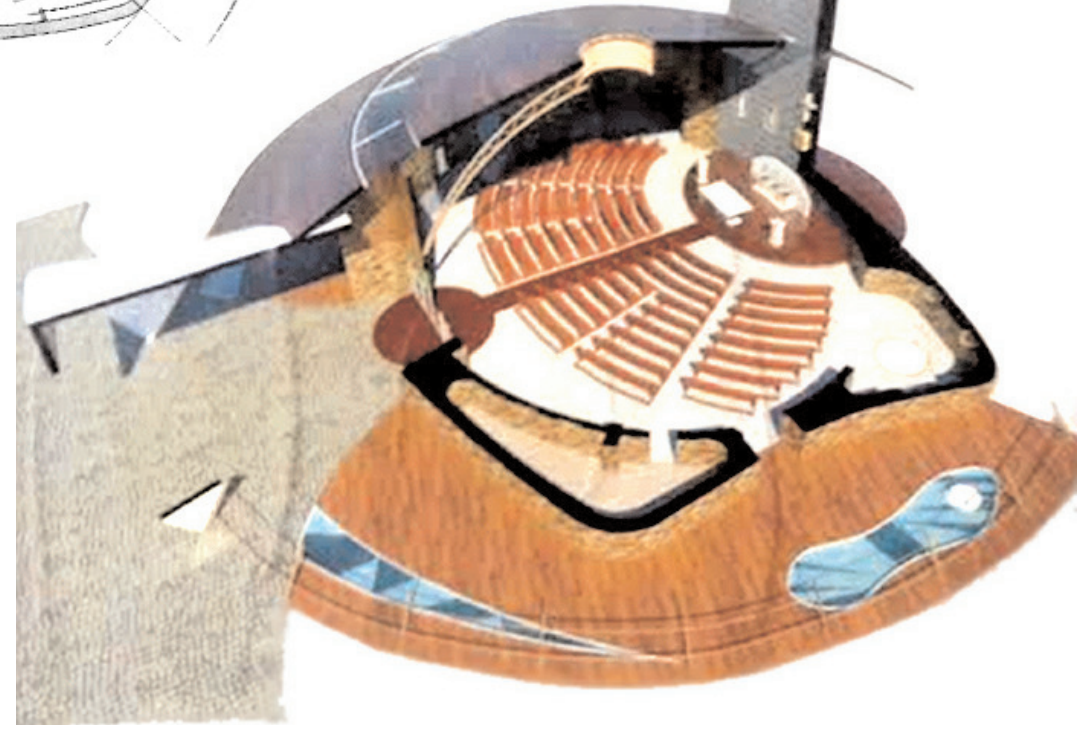



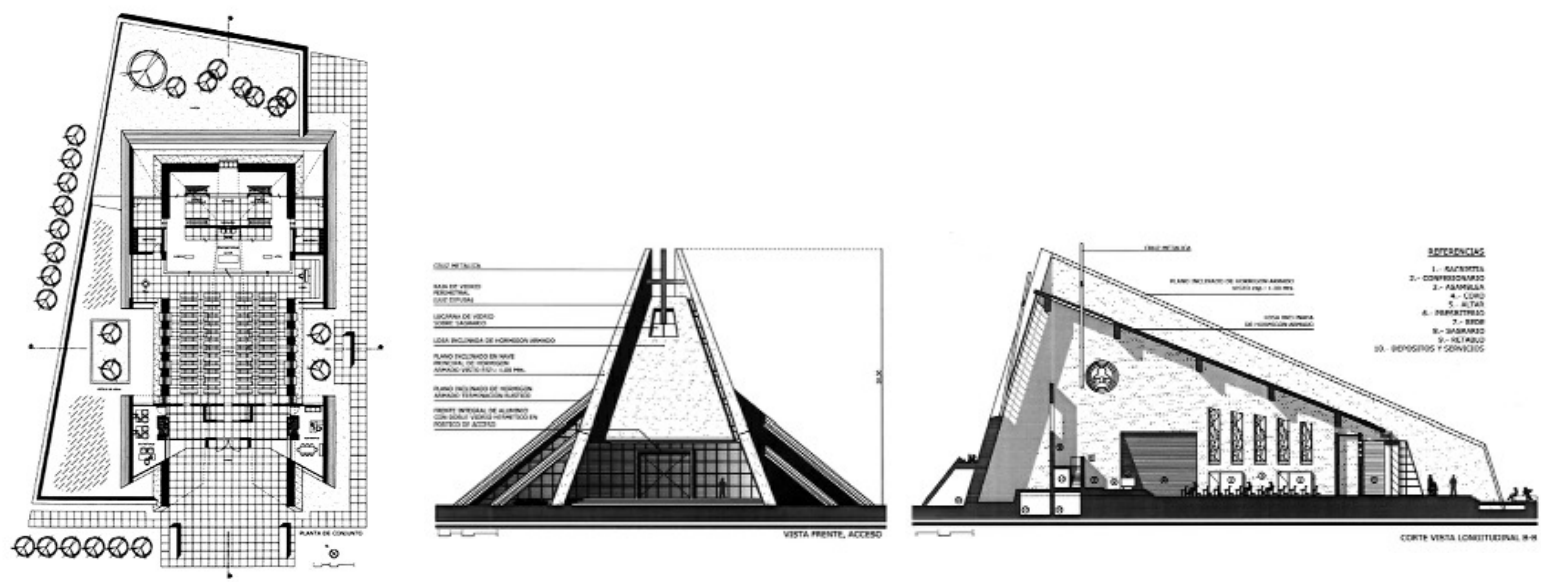

Fig. 10. Darío Gutis, anteproyecto para una iglesia en Buenos Aires, 2010; planta, alzado principal y corte longitudinal.

\section{EXPERIENCIAS DE LA CÁTEDRA}

En sus comienzos $-\mathrm{y}$ teniendo en cuenta que las horas cátedra eran una vez por semana-, se optó por dar clases teóricas, las cuales trataban de inculcar en los alumnos la importancia de asistir a la iglesia, ya que no todos son necesariamente católicos. Para ello, se recordaban las distintas funciones que ofrecía la liturgia a través de los tiempos y los distintos estilos arquitectónicos, la finalidad y ubicación de los sacramentos, los elementos del presbiterio, la sacristía y secretaría, etc. Mediante análisis de iglesias en diferentes diócesis, se pudo observar el impacto del edificio en el contexto urbano y las experiencias espaciales que surgían debido a las diferentes volumetrías.

Ya que una de las intenciones primarias de la cátedra era lograr que el tema iglesia fuera un hecho arquitectónico a tener en cuenta en la Facultad de Arquitectura como una síntesis de varias materias, en el programa de los años posteriores se optó por desarrollar un anteproyecto de una iglesia parroquial.

Cada curso presenta distintas asimetrías en sus conocimientos de doctrina y conceptos sobre el hacer arquitectónico. Asimismo, cada corrección ofrece la oportunidad de recordar distintos aspectos de la doctrina cristiana, desde la presencia real de su Fundador, hasta los sacramentos instituidos, la liturgia de la Palabra y los comentarios sobre el Antiguo y el Nuevo
Testamento, el tema de la fe, y otros aspectos que surgen a partir del trato coloquial con los alumnos. Esto permite resolver tanto cuestiones de diseño — desde una abertura hasta el tratamiento del solado- hasta cuestiones cotidianas que se refieren a la vida universitaria y religiosa personal. Algunos ejemplos de trabajos de los alumnos, tratan de reflejar estos intentos (Fig. 08-10).

Proponemos el esfuerzo de tratar de vivir el Evangelio, no sólo como un hecho histórico, sino como la necesidad de interpretarlo en la vida actual, debido a su vigencia y beneficio. Entonces el proceso intelectual que toca al diseño del edificio, va acompañado de la razón de ser del mismo.

Sin el correspondiente proceso, entendemos que no podemos tener un juicio de valor sobre el mismo, y aconsejamos a los alumnos que deben tomar más tiempo para reforzar y optimizar su anteproyecto, en el caso de no alcanzar un nivel aceptable.

\section{BIBLIOGRAFÍA}

Conferencia Episcopal Argentina. 1993. Lineamientos generales para la construcción de iglesias. Buenos Aires: Oficina del libro.

\section{PROCEDENCIA DE LAS ILUSTRACIONES}

\section{Archivo UCALP.}

